\title{
Laparoscopic Management of
}

\section{Rectal Tumors in Developing Countries}

Iqbal Saleem Mir, Suhail Nazir, Tajamul Rashid, Hamid Raashid*, Farooq Ahmad Reshi,

\author{
Mudasir Hajini, Sikander Lone \\ Department Of General Surgery, Government Medical College, Srinagar
}

Received: June 06, 2017; Accepted: January 10, 2018; Published: January 25, 2018

*Corresponding author: Hamid Raashid, Department Of General Surgery, Government Medical College, Srinagar, E-mail: drraashidhamid@ gmail.com

\begin{abstract}
Aims: The laparoscopic surgery for rectal cancer is much more challenging than laparoscopic colonic surgeries due to limited space in pelvis. However its feasibility and safety has been established in many randomised and non-randomised studies. The aim of our study was to evaluate the short term outcomes of laparoscopic approach for rectal cancer surgeries in developing countries.
\end{abstract}

Methods: 30 patients were included in our prospective observational study out of which 14 underwent laparoscopic and 16 underwent open surgeries for rectal cancer. Short term outcomes in both groups were recorded and analysed. Conversions were excluded from the study.

Findings: The intra operative blood loss $(146.7+25.3 \mathrm{ml}$ in laparoscopic group (LAP) and $353.8+32.2 \mathrm{ml}$ in open surgical (OS); $p$-value $<0.001)$, requirement of analgesia $(4.1+2.3$ doses in LAP and $8.4+3.1$ doses in OS group: $p$-value $<0.0002)$, time of resumption of intestinal function (i.e. appreciation of flatus after 46.8 +6.2 hours in LAP group and82.9 + 8.1 hours in OS group; $p$-value $0.001)$ and post-operative hospital stay $(6.5+1.3$ days in LAP group and $9.1+2.1$ days in OS group; $p$-value $<0.0002$ ) were relatively less in laparoscopic group. The early post-operative complications $(p$-value $=0.260)$ and lymph node yield $(13.6+2.5$ in LAP and $14.3+$ 2.1 in OS group; $p$-value $=0.412$ ) were comparable in the two groups. However, operative time was $205.5+21.3$ minutes in LAP group and $151.1+17.8$ minutes in OS group ( $p$-value $<0.001)$. No short term mortality was noted.

Interpretation: Laparoscopic surgery for rectal tumors is feasible, oncologically safe and has better short term outcomes.

Keywords: Colorectal Carcinoma; Rectal Cancer; Low Anterior Resection; Abdominoperineal Resection; Total Mesorectal Excision;

\section{Introduction}

Colorectal cancer is very significant surgical problem worldwide. Approximately one million people per year develop this tumor and more than half of them will die of this malignancy [1]. Colorectal cancer is the third most common cancer in Kashmir in both men and women [2]. In our valley the incidence of carcinoma rectum per 100000 is 1.82 in males and 1.71 in females (as per 2009 statistics) with highest incidence in district Srinagar and lowest in district kupwara [3].This represents an enormous challenge and creates huge interest especially with systemic adjuvant and palliative treatment aiming to improve and prolong survival. Adjuvant therapy, such as chemotherapy and radiotherapy, can improve survival in colorectal cancer patients. However, the only treatment with curative intent is surgical resection of the tumour. Currently, over $90 \%$ of colorectal cancers are treated surgically [4]. Laparoscopy is quite advantageous in benign abdominal conditions and offers less blood loss, early return of bowel function, reduced pain, and shorter hospital stay decreased disability and better cosmesis [5]. But in the scenario of malignancy, these advantages are of secondary importance. The basic issue of oncologic feasibility, port site recurrence and safety of laparoscopic resection have been concerning and questions were raised regarding adherence to oncologic principles during laparoscopic surgery $[6,7,8]$, but the dust has settled now and laparoscopy has gained world-wide acceptance for being oncologically safe if strict technical adherence to oncologic principles is exercised $[9,10]$. We have made an endeavour to critically evaluate laparoscopic rectal resection in malignant disorders and assess its safety and feasibility along with analysis of short term outcomes.

\section{Material and Methods}

Thirty patients were included in our study out of which 14 (8 male, 6 female) underwent laparoscopic and 16 (9male, 7 female) underwent open surgery for rectal cancer from 0103-2015 to 31-03-2016. The study was conducted in the post graduate department of general surgery Government Medical College, Srinagar. The patients were aged 18 years or above and were eligible for curative resection of rectal cancer observed at sigmoidoscopy or colonoscopy and confirmed by biopsy. The patients with metastatic disease, previous rectal surgery, and acute intestinal obstruction, synchronous need for other abdominal surgery and those with absolute contraindication to general anaesthesia were excluded from the study.

All patients were evaluated and accessed according to pre formed pro forma including elaborate history, detailed clinical examination, base line investigations and specific investigations like serum carcinoembreyonic antigen (CEA), ultrasonography of 
abdomen and pelvis, sigmoidoscopy/ colonoscopy with biopsy and histopathology. Pre-operative Computerised Tomography (CT) scan and Magnetic Resonance Imaging (MRI) of pelvis were done as staging investigations. Patients were given free choice to undergo laparoscopic or open resection and a written informed consent was taken in each case. The pre-operative preparations were standardise in both laparoscopic and open group. Assessment of resectibility was done on the basis of preoperative imaging; however, final decision on resectibility was taken at the time of laparoscopy/laparotomy. The patients were categorized into anterior resection (AR), low anterior resection (LAR) and abdominoperineal resection (APR) based on the distance of tumor from anal verge. However, this grouping was not hard and fast but some sort of flexibility was exercised more so for low rectal cancers whenever sphincter preservation was found to be feasible but not at the cost of onchologic safety.

Laparoscopic procedure was done after creating pneumoperitoneum using carbondioxide insufflations by percutaneous veress or by open hasson's technique and intraabdominal pressure of 12-15 mm Hg was established. Diagnostic laparoscopy was done to access the disease. This was followed by insertion of multiple ports and working instruments under vision. Mobilisation of rectum was done using harmonic shears or monopolar cautery. Critical structures viz. ureters, hypogastric nerves and pelvic parasympathetic plexus were protected and vascular pedicles were ligated/ clipped. In each case total mesorectal excision or tumour specific mesorectal excision was performed in an appropriate plane. Distal end of the mobilised tumor was resected intracorporaly and growth was exteriorised using double glove technique through a small incision around 4 $\mathrm{cm}$ in length in left lower quadrant and divided with appropriate proximal clearance. In case of stapler anastomosis the proximal end anvil was placed extracorporealy, laparotomy closed and anastomosis was performed intracarporealy by circular stapler introduced per rectum. Colorectal anastomosis was checked by hydropneumatic test and drain was kept in pelvis. In case of abdominoperineal resection laparoscopic procedure was followed by perineal resection in standard fashion and permanent colostomy in left lower quadrant of abdomen. Perineal surgeon mobilised rectum and whole mesorectum and specimen were retrieved via perineum. Perineal wound was closed and low negative suction drains kept inside. Open surgery was done as per the established techniques confirming to the standard rules of rectal cancer resection. Protective ileostomy was not performed in any patient.

The post-operative course including all complications was documented. Short term outcomes like amount of blood loss, operative time, requirement of analgesia, resumption of intestinal function, duration of hospital stay were properly analysed. Histopathologic review of the resected specimen was done and margin status, distance of growth from distal margin and lymph node status were analysed.

\section{Results}

A total of 30 patients were operated in our study out of which 14 underwent laparoscopic and 16 underwent open surgical resection for biopsy proven rectal malignancy from 01-03-2015 to 31-03-2016. The patient parameters and sex ratio were comparable in the laparoscopic and open group. In the laparoscopic group 5 underwent AR, 4 underwent LAR and 5 underwent APR while in open group, 5 patients underwent AR, 5 underwent LAR and 6 underwent APR. 8 laparoscopically operated (LAP group) patients had positive lymph nodes while as 10 open group patients had positive lymph nodes.

Table 1: Patient parameters in LAP and open group.AR (Anterior Resection), LAR ( Low Anterior Resection), APR (Abdominoperineal resection)

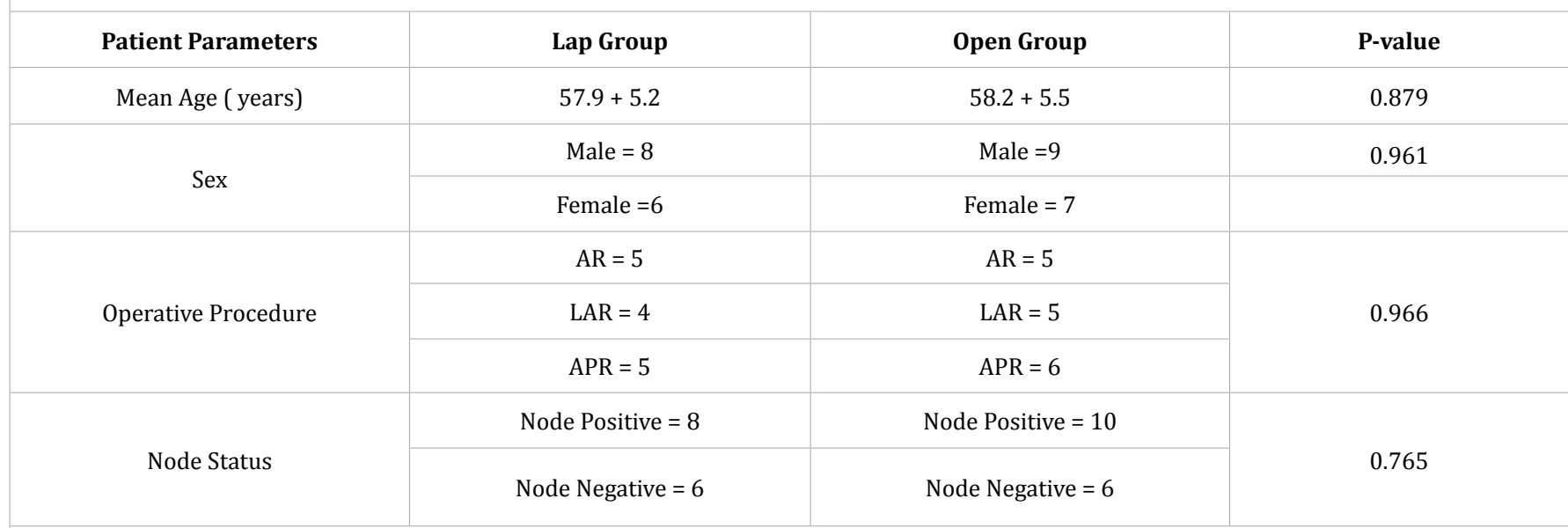

The operative time for laparoscopically resected patients was more; however, intra operative blood loss was significantly low. The laparoscopically operated patients appreciated flatus earlier and orals could be started earlier. Post-op hospital stay in laparoscopically operated patients was shorter $(6.5 \pm 1$ days $)$ compared to open group. 
Table 2: operative outcomes in LAP and open group

\begin{tabular}{|c|c|c|c|}
\hline Operative Outcomes & Lap Group & Open Group & P-Value \\
\hline Operative Time & $205.5+21.3$ minutes & $151.1+17.8$ minutes & $<0.001^{*}$ \\
\hline Intra operative blood loss & $146.7+25.3 \mathrm{ml}$ & $353.8+32.2 \mathrm{ml}$ & $<0.001^{*}$ \\
\hline No. of doses of parental analgesics required & $4.1+2.3$ doses & $8.4+3.1$ doses & $0.0002^{*}$ \\
\hline No of patients requiring blood transfusion & Intra-op $=4$ & Intra op $=8$ & 0.411 \\
\cline { 2 - 4 } & Post-op $=2$ & Post op $=6$ & 0.226 \\
\hline Passing flatus & $46.8+6.2$ hours & $82.9+8.1$ hours & $<0.001^{*}$ \\
\hline Oral intake & $58.3+8.3$ hours & $96.7+10.2$ hours & $<0.001^{*}$ \\
\hline Post-op hospital stay & $6.5+1.3$ days & $9.1+2.1$ days & $0.0002^{*}$ \\
\hline
\end{tabular}

\section{*Statistically Significant Difference (P-value $<0.05)$}

The pathologic review of the resected specimen was quite comparable in the two groups and has been summarized in table 3 . The specimen length for different operations in two groups was comparable. Lymph node yield in laparoscopic group was $13.6+2.5$ and that in open group was $14.3 \pm 2.1$. Distal resection margin of LAR specimen was $4.1 \pm 1.2 \mathrm{~cm}$ from the growth in Laparoscopic LAR while it was $4.5 \pm 2.4 \mathrm{~cm}$ in open LAR

Table 3: Pathologic Review of laparoscopically 9.7 resected and open specimen

\begin{tabular}{|c|c|c|c|}
\hline Pathologic Review & Lap Group & Open Group & P-value \\
\hline \multirow{3}{*}{ Specimen length ( centimetres) } & $\mathrm{AR}=18.2+3.3 \mathrm{~cm}$ & $\mathrm{AR}=18.5+4.2 \mathrm{~cm}$ & 0.723 \\
\hline & $\mathrm{LAR}=18.3+2.1 \mathrm{~cm}$ & $\mathrm{LAR}=18.7+3.4 \mathrm{~cm}$ & 0.706 \\
\hline & $\mathrm{APR}=25.2+3.6 \mathrm{~cm}$ & $\mathrm{APR}=26.1+4.4 \mathrm{~cm}$ & 0.548 \\
\hline Lymph Node Yield & $13.6+2.5$ & $14.3+2.1$ & 0.412 \\
\hline
\end{tabular}

Table 4: complications noted in laparoscopic and open resection group

\begin{tabular}{|c|c|c|c|}
\hline Complications & Lap Group & Open Group & P-value \\
\hline Intra operative bleeding & Nil & 2 & 0.485 \\
\hline Rectal injury & 1 & Nil & 0.467 \\
\hline Urinary bladder injury & Nil & Nil & - \\
\hline Prolonged ileus & Nil & 2 & 0.485 \\
\hline Urinary retention & Nil & 1 & 1 \\
\hline Anastomotic leak & 1 & Nil & 0.467 \\
\hline Intra-abdominal abscess & Nil & Nil & - \\
\hline Wound infection & 1 (perineal) & 2 & 1 \\
\hline
\end{tabular}

Post op and intra op complications were documented and compared. Rectal injury was encountered in one patient during laparoscopic resection and anastomotic leak in one patient who had undergone laparoscopic LAR which was managed conservatively. Intraoperative bleeding occurred in 2 open group patients who required multiple blood transfusions. Wound infection occurred in 2 patients in open group while it was observed in 1 perineal wound in laparoscopic group. Urinary retention occurred in 1 patient in open group and postop ileus in 2 patients in open group. Overall there was no in hospital mortality, defined as death within 30 days after surgery. 


\section{Discussion}

The use of laparoscopic approach for colorectal surgery started and advanced over last 2 decades since the first laparoscopic colonic resection by Jacob $1991[11,12]$. The first report of comparing open vs laparoscopic approach for rectal cancer came from a subset analysis of CLASSIC trial, which demonstrated comparable short term results [13]. COREAN trial is one of the largest trials comparing laparoscopic rectal resection with open [14]. Laparoscopic surgery had advantages of fast recovery, minimized postoperative ileus and pain, shorter hospital stay and rapid recovery $[15,16]$. However, early concerns and controversies regarding laparoscopic resection were raised questioning the oncologic adequacy, port site metastasis, safety, tumor localization and conversion.

The oncologic adequacy regarding laparoscopic resection centred on the possibility of TME with this approach. Interestingly, it has been found that laparoscopy is not only equivalent but superior in a univariate analysis of long term survival [17]. This could be explained by better preparation of TME facilitated by magnification of endoscopic camera [18]. Recently published review about laparoscopic TME also indicated short term advantages of laparoscopic TME compared to open TME [19]. Fixation of trocar to abdominal wall, high vascular ligation, isolation of specimen before extraction from abdominal cavity and intraperitoneal and trocar site irrigation with tumoricidal solution have been described as routine to avoid port site metastasis [20]. The port site metastasis has not been a significant issue in presence of adequate training and laparoscopic skills [21, 22]. Zmorareported port site recurrence rate of $1 \%$ in a review of 1737 patient's undergone laparoscopic colorectal resection for malignancy [23].

The main concern regarding the safety was leak especially in low anterior resection and injury to critical structures. However, the leak rate in laparoscopic resection group has been reported to be comparable to open resection group (less than 10\%) [24, 25]. Furthermore, laparoscopic magnified view allows better identification of critical structures and hence less chances of injury [18]. We routinely checked the anastomosis for any leak by hydro pneumatic insufflations test. Colonoscopic tattooing with Indian-ink was done preoperatively in 2 patients with small (less than $2 \mathrm{~cm}$ lesion) lesions for better localization.

Appropriate intraoperative judgement as when to convert to an open procedure is also critical to the safe adoption of laparoscopic approach. It is important to identify the need to convert as soon as possible so as to reduce operative time and overall cost. The rate of conversion has been reported to be around $15 \%$. Main reasons being difficulty to provide exposure or to identify anatomy, fixity of tumor to adjacent structures and complications arising from long term pneumoperotoneum [26]. Converted patients were excluded from our study.

The operative time of laparoscopic resection group in our study was longer compared to open resection group, however, with increasing experience operative time could be reduced. The advantages of laparoscopic resection like less blood loss, less postop pain, early appreciation of flatus and initiation of orals helping in short hospital stay observed in our study were consistent with the results of published studies [5, 17]. Pathological outcomes were also comparable in both lap and open resection groups. However, higher lymph node yield in open resection group could be attributed to extensive dissection. The complication rate of laparoscopic resection group was overall lower compared to open resection group with less incidence of intraoperative bleeding, prolonged ileus, urinary retention, wound infection in the former. These results have been confirmed by many authors.27,28Though no long term follow up was done in our study but till date all randomized and non-randomized studies have shown no significant difference in long term outcomes of laparoscopic and open resection for rectal cancer with added less morbidity laparoscopically operated patients [5, 17].

\section{Conclusion}

Laparoscopic resection of rectal malignancy is surgically safe and oncologically feasible. It carries the additional benefits of being minimally invasive like less pain, less blood loss, early return of bowel function, and early resumption of orals, shorter hospital stay and early return to work. Even though, laparoscopic surgery takes longer time but it provides the above mentioned short term benefits. The cost of the laparoscopic surgery is quite compensated by decreased hospital stay and early return to work. The conversion rates of laparoscopic procedure are quite acceptable and with increasing surgical experience it is expected to drop further. The oncologic feasibility of laparoscopic procedure for rectal surgery has been established by number of randomised and non-randomised studies. This observation is very significant because in case of laparoscopic malignancy oncologic safety is the primary concern. As the laparoscopic surgery has added benefits of favourable short term outcomes and is oncologically effective, days are not far when laparoscopy becomes standard of care for rectal malignancies.

\section{References}

1. Parkin DM, Dray F, Ferlay J, Paola Pisani. Global Cancer Statistics 2002. CA Cancer j clin. 2005;55(2): 74-108. DOI: 10.3322/canjclin.55.2.74

2. Sameer AS, ul Rehman S, Pandith AA, Chowdhri NA, Wani KA, Siddiqi MA et al. Molecular gate keepers succumb to gene aberrations in colorectal cancer in Kashmiri population, revealing a high incidence area. Saudi J Gastroenterol. 2009;15(4):244-52. doi: 10.4103/1319-3767.56102

3. Javaid G, Zargar SA, Rather S, Abdul Rashid Khan, Bashir Ahmad Khan, Ghulam Nabi Yattoo et al. Incidence of colorectal cancer in Kashmir valley, India. Indian Journal of Gastroenterology. 2011;30(1):7-11.

4. Beart RW, Steele GD Jr, Menck HR, Chmiel JS, Ocwieja KE, Winchester DP. Management and survival of patients with adenocarcinoma of colon and rectum: a national survey of the Commission on Cancer. 1995;81(3):225-36.

5. Wen- Xi W, Yao-Min S, Yi-Bin H. Laparoscopic Versus Conventional Open Resection of Rectal Carcinoma: A Clinical Comperative Study. World Journal of Gastroenterology. 2004;10(8):1167-1170. doi: 10.3748/ wjg.v10.i8.1167 
6. Cirocco WC, Schwartzman A, Golub RW. Abdominal Wall Recurrence after Laparoscopic Colectomy For Colon Cancer. Surgery 1994;116(5): 842-846.

7. Waxner SD, Cohan SM. Port site Metastasis after Laparoscopic Colorectal Surgery for Cure of Malignancy. British Journal of Surgery 1995;82:295-298. DOI: 10.1002/bjs.1800820305

8. Lacy AM, Delgado S, Garcia Val de cas JC, A Castells, JM Piqué, L Grande, et al. Portsite Metastasis And Recurrence After Laparoscopic Colectomy: A Randomised Trail. Surg. Endosc. 1998;12(8):1039-1042.

9. Slanetz C A Jr. Effects of no Touch Isolation on Survival and Recurrence in Curative Resection Of Colorectal Cancer. Ann SurgOncol. 1998;5(5):390-398.

10.Heald RJ, Husband EM, Ryall RD. The MesorectumIn Rectal Cancer Surgery: The Clue to Pelvic Recurrence. British Journal of Surgery 1982;69(10):613-616.

11.Cooperman AM, Katz V, Zimmon D. Laparoscopic Colon Resection: A Case Repot. LaproEndoscSurg. 1991;1:221-224.

12.Saclarides T J, Ko S T, Airan MC, C Dillon J Franklin. Laparoscopic Removal Of a Large Colonic Lipoma: A Repot Of a Case. Dis Colon Rectum .1991;34(11):1027-1029.

13.Jayne DG, Guillon PJ, Thrope H, Philip Quirke, Joanne Copeland, Adrian MH Smith, et al. Randomised Trail Of Laparoscopic Assisted Resection Of Colorectal Carcinoma.3 year results of the UK MRC CLASSIC group. J ClinOncol. 2007;25:3061-3068. DOI: 10.1200/JC0.2006.09.7758

14.Kang S B, Dark J W, Jeong S Y, et al.Open Versus Laparoscopic Surgery For Mid or Low Rectal Cancer After Neoadjuvanct Chemo radiation Therapy ( COREAN trail): Short Term Outcomes Of Open Label RCT. Lancet Oncol. 2010;11(7):637-645. Doi: 10.1016/S14702045(10)70131-5

15.Manson J R, Darzi A, Carey PD, PJ Guillou FRCS. Prospective Evaluation Of Laparoscopic Assisted Colectomy In an Unselected Group Of Patients. Lancet. 1992;340:831-833. Doi.org/10.1016/0140-6736(92)92694-B

16.Yamamoto S, Watanabe M, Hasegawa $\mathrm{H}$, et al. Prospective Evaluation of Rectal Surgery for Recto sigmoid and Rectal Carcinoma. Dis of Colon and Rectum 2002;45:1648-1654.

17.Michael AS, Klaus Uwe G, Karl Walter J, Markus M. Heiss. Comparison Of Laparoscopic Versus Open Access Surgery inPatients With Rectal Cancer : A Prospective Analysis. Diseases of Colon And Rectum 2008;51(4):385-391.
18.Marecau XJ, Rubino F, Leroy J. Laparoscopic TME for rectal cancer surgery. Dig Dis. 2005;23:135-141.

19.Breukink S, Pierie J, Wiggers F. Laparoscopic versus Open Total Mesorectal Excision for Rectal Cancer. Cochrane Database System review. 2006;CD005200. DOI: 10.1002/14651858.CD005200

20.Balli JE, Franklin ME, Almeda JA, JL Glass, JA Diaz, M Reymond. How To Prevent port Site Metastasis In Laparoscopic Colorectal Surgeries. Surgical endosc. 2000;14(11):1034-1036.

21.COLOR Study Group. COLOR- A Randomized Clinical Trial Comparing Laparoscopic and Open Resection for Colon Cancer. Dig Surg. 2000;17(6):617-622.

22.Lumley J, Stitz R, Stevenson A, Fielding G, Luck A. Laparoscopic Colorectal surgery for Cancer: Intermediate to longterm Outcomes. Dis Colon Rectum. 2002;45(7):867-872.

23.Zmora 0, Gervaz P, Wexner SD. Tocar Site Recurrence in Laparoscopic Surgery For Colorectal Cancer. Surg Endosc. 2001;15(8):780-793.

24.Pera M, Delgado S, Garcia val de casas J C. The Management Of Leaking Rectal Anastomosis By Minimally Invasive Technique. Surg Endosc. 2002;16(4):603-606.

25.Scheidbach H, Schneider C, Hulgel O, Bärlehner E, Konradt K, Wittekind C, et al. Laparoscopic Sigmoid Resection for Cancer: Curative Resection and Preliminary Medium Term Results. Dis Colon Rectum. 2002;45(12):1641-1647.

26.Gervaz P, Pikarsky A, Ulech M, M SecicJ, Efron B. Belin et al. Converted Laparoscopic Colorectal Malignancy. Surg Endosc. 2001;15(8):827832.

27.Weeks Jc, Nelson H, Gellber S, Sargent D, Schroeder G; Clinical Outcomes of Surgical Therapy (COST) Study Group. Short Term Quality Of Life Outcomes Following Laparoscopic Assisted Colectomy versus Open Colectomy For Colon Cancer- A Randomised Trail. JAMA. 2002;287(3):321-328.

28.Wu FP, Sietses C, Von Blomberg BM, van Leeuwen PA, Meijer S, Cuesta MA. Systemic And Peritoneal Inflammatory Response After Laparoscopic Or Conventional Colon Resection In Cancer Patients: A Prospective Randomised Trail. Dis Colon Rectum. 2003;46(2):147-155. 